\title{
Cellular and molecular insights on inflammatory immune response regulation during acute aspergillosis in chicken and turkey poults
}

Vahsen, T. ${ }^{1}$, Zapata, L. ${ }^{1}$, Guabiraba, R. ${ }^{2}$, Melloul, E. ${ }^{1}$, Guibert, M-J. ${ }^{3}$, Botterel, F. ${ }^{1}$, Guillot, J. ${ }^{1}$, Arné, P. ${ }^{1}$, Risco-Castillo, V. ${ }^{1}$

${ }^{1}$ Dynamyc research group EA 7380, Ecole Nationale Vétérinaire d'Alfort, UPEC, Maisons-Alfort, F94700, France

2 ISP, INRA, Université François Rabelais de Tours, Nouzilly, France.

${ }^{3}$ Lohmann France, Z.A. du Grand Moulin - 85250 Saint-Fulgent, France.

\section{Summary.}

Avian aspergillosis produced by Aspergillus fumigatus is a global threat in terms of animal health and husbandry. Susceptibility to this fungal infection is host species-dependant, and poultry such as turkey poults, can show high morbidity and mortality rates. However, little is known regarding the features of avian immune response after inhalation of Aspergillus conidia and no information regarding inflammatory pathways has been so far described. The objective of this study was to improve the understanding of the interactions between $A$. fumigatus and local immune cells and the Th1-like immune response by comparing two avian models of acute aspergillosis.

\section{Experimental acute respiratory aspergillosis.}
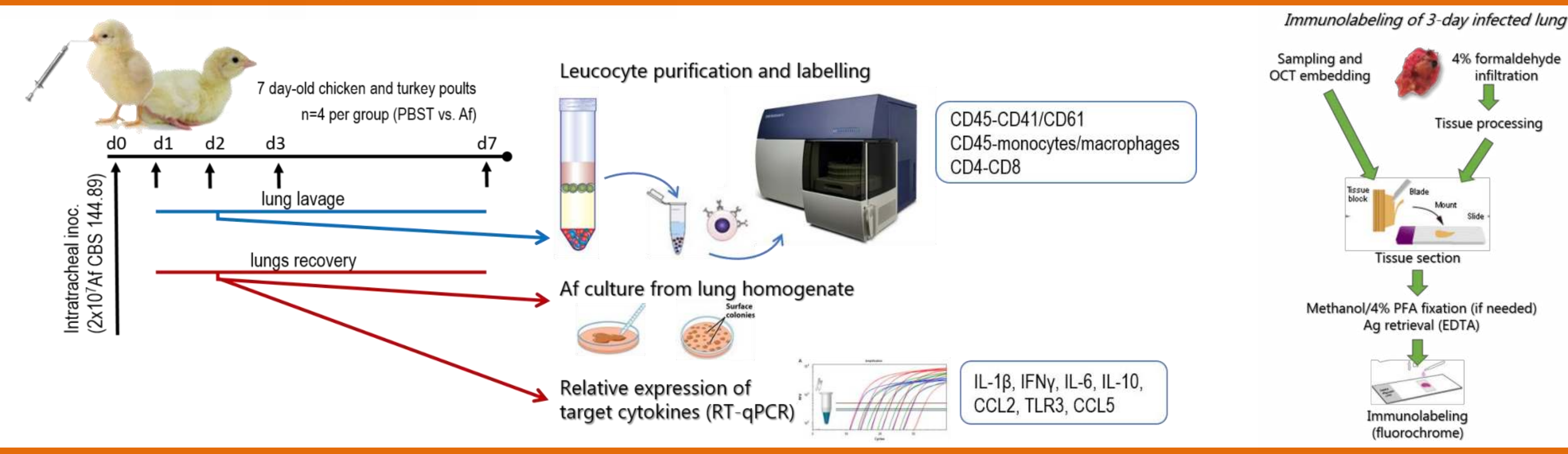

\section{Results.}

Higher proportion of leucocytes in chicken lung lavage were found (Fig. 1). Chicken thrombocytes increased after infection (recruitment and infiltration in lung tissue) (Figs. 2 and 3 ). No detectable differences regarding monocytes, CD4+ and CD8+ $T$ cells were observed.

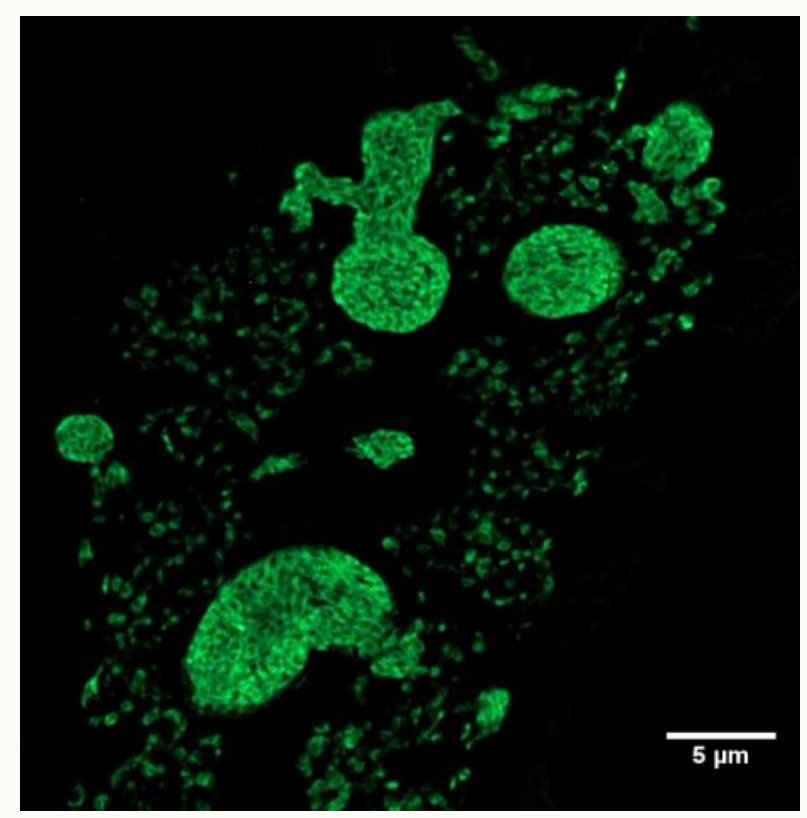

Fig. 3 Chicken CD41+ thrombocytes clustered in likely $A$. fumigatus lung granulomas
Fig 1. Leucocytes in lung lavage

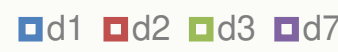

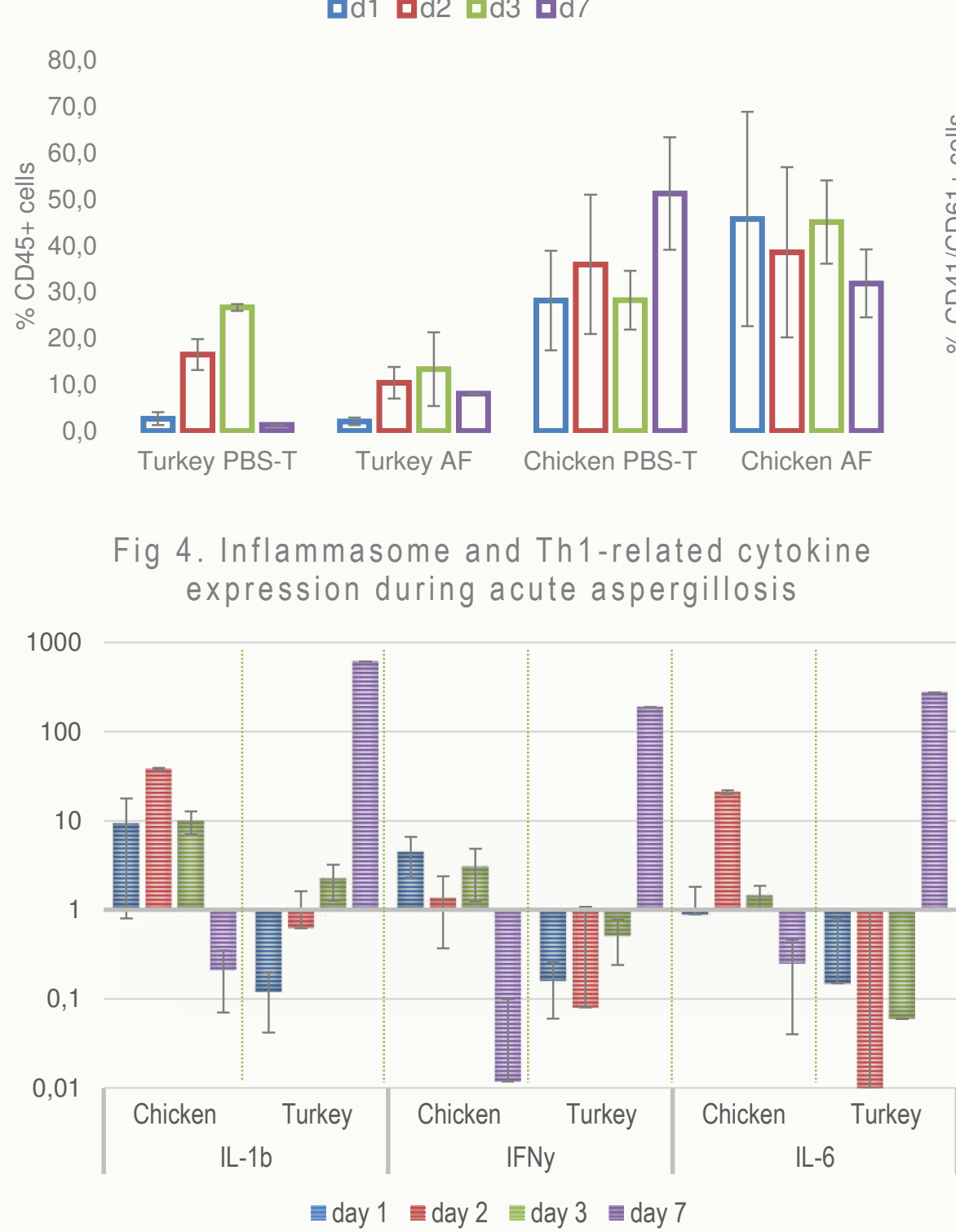

Fig 2. Thrombocytes in lung lavage 口d1 $\mathbf{D d} 2 \mathrm{~d} 3 \mathbf{\square d} 7$

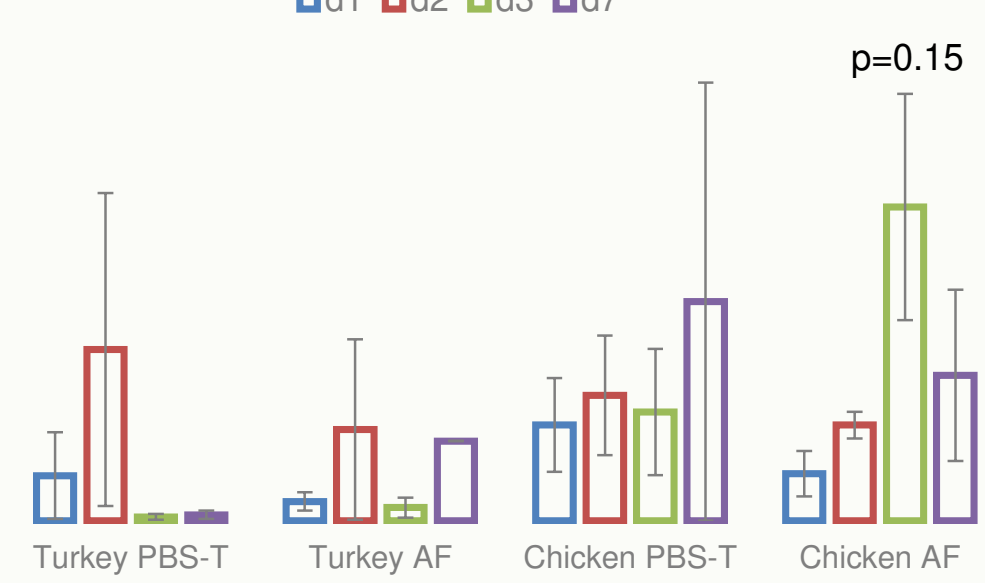

Fig 5. Th2-related cytokine and chemokine expression during acute aspergillosis

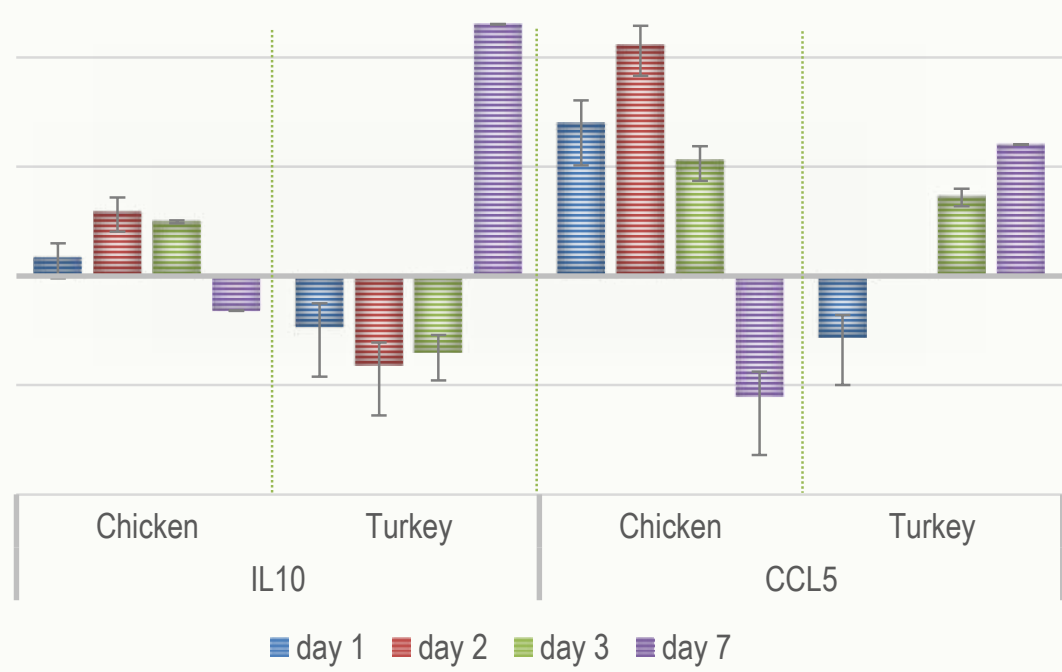

Early expression of inflammasome-related IL-1 predicts efficient Th1-like response together with heterophils and thrombocytes recruitment in chickens (Fig. 4). It is confirmed by IFNg and IL-6 expression, while in turkeys they seem to be activated late after infection. On the hand, disproportionate turkey IL-10 expression could overstimulate Th2-like response and risk macrophages and heterophils recruitment (Fig. 5).

\section{Conclusion.}

Our results show for the first time local immune response differences on two avian species with different sensibility to Aspergillus infection. They confirm the role of a Th1-like response to ensure the establishment of cellular immune response and suggest an important participation of phagocytic thrombocytes during infection. 